\title{
NON-UNIFORM DECAY OF MHD EQUATIONS WITH AND WITHOUT MAGNETIC DIFFUSION
}

\author{
RUBÉN AGAPITO AND MARIA SCHONBEK*
}

\begin{abstract}
We consider the long time behavior of solutions to the magnetohydrodynamics equations in two and three spatial dimensions. It is shown that in the absence of magnetic diffusion, if strong bounded solutions were to exist their energy cannot present any asymptotic oscillatory behavior, the diffusivity of the velocity is enough to prevent such oscillations. When magnetic diffusion is present and the data is only in $L^{2}$, it is shown that the solutions decay to zero without a rate, and this nonuniform decay is optimal.
\end{abstract}

\section{INTRODUCTION}

We consider the Magnetohydrodynamics equations (MHD) in two and three dimensions. We deal with questions regarding long time behavior of solutions to the MHD with and without magnetic diffusion. The MHD equations model the interactions between a magnetic field and a viscous incompressible fluid of moving electrically charged particles.

In non-dimensional form the equations can be expressed by

$$
\begin{aligned}
\frac{\partial}{\partial t} u+(u \cdot \nabla) u-S(B \cdot \nabla) B+\nabla\left(P+\frac{S}{2}|B|^{2}\right) & =\frac{1}{\operatorname{Re}} \Delta u, \\
\frac{\partial}{\partial t} B+(u \cdot \nabla) B-(B \cdot \nabla) u & =\delta \Delta B, \\
\nabla \cdot u & =0, \nabla \cdot B=0, \\
u(x, 0) & =u_{0}(x), B(x, 0)=B_{0}(x),
\end{aligned}
$$

where $u=u(x, t)=\left(u^{1}(x, t), u^{2}(x, t), \ldots, u^{n}(x, t)\right), B=B(x, t)$ and $P=P(x, t)$ denote the unknown velocity, the magnetic field and pressure of the fluid at a point $(x, t) \in \mathbb{R}^{n} \times \mathbb{R}_{+}$, respectively. The term $\frac{|B|^{2}}{2}$ denotes magnetic pressure. The positive constants appearing in the equations are Re, the Reynolds number, Rm, the magnetic Reynolds number, and $S=M^{2} /(\operatorname{Re} \mathrm{Rm})$, where $M$ is the Hartman number. For the sake of notational simplicity, and with minor loss of generality, we set all these constants equal to one. After rescaling $u$ and $B$, let $p=P+\frac{1}{2} S|B|^{2}$

The work of M. Schonbek was partially supported by NSF grant DNS-0600692. 
denote the total pressure, Equation (1.1) can be rewritten as

$$
\begin{aligned}
\frac{\partial}{\partial t} u+(u \cdot \nabla) u-(B \cdot \nabla) B+\nabla p & =\Delta u, \\
\frac{\partial}{\partial t} B+(u \cdot \nabla) B-(B \cdot \nabla) u & =\delta \Delta B, \\
\nabla \cdot u & =0, \nabla \cdot B=0, \\
u(x, 0) & =u_{0}(x), B(x, 0)=B_{0}(x) .
\end{aligned}
$$

The initial data $\left(u_{0}(x), B_{0}(x)\right)$ will be chosen below in appropriate spaces. Derivations of these equations can be found in Cha81, Cow76, LLP84.

Many authors have studied MHD equations from the point of view of existence and long time behavior. Without making a complete list of all authors we would like to mention some of the relevant literature. In particular important results on existence were obtained, among others, in [Koz87, HX05b, HX05a, Wu02]. In the direction of decay interesting results can be found in the papers MS89, Kim02, SSS96. The methods used for decay in [MS89] were based on Fourier Splitting [Sch85]. The paper Kim02 uses Gevrey regularity and it is based on ideas developed by Foias and Temam. Similar methods were used for the Navier-Stokes equations and can be found in OT00.

Several questions will be addressed regarding the long time behavior of the solutions. In this regard, in the absence of magnetic diffusion, we are going to analyze the following problem.

Is the diffusion introduced by the velocity alone sufficient to prevent compensatory oscillations? Specifically, simple calculations shows that the following energy inequality holds when $\delta=0$,

$$
\frac{d}{d t}\left(\|u(t)\|_{2}^{2}+\|B(t)\|_{2}^{2}\right) \leq-2\|\nabla u(s)\|_{2}^{2} .
$$

This shows that the combined energy decays, but allows the possibility of separate oscillations in $\|u\|_{2}$ and $\|B\|_{2}$ that could compensate each other. In this paper we show that in the case that there were to exist strong solutions which are bounded such oscillations can never occur. Specifically it is shown that

Theorem 1. Let $n=3$. Suppose that there exists $(u, B)$ a strong bounded solution to the MHD equations with $\delta=0$, magnetic field bounded in $L^{\infty}$, and data $\left(u_{0}, B_{0}\right) \in\left(L^{1} \cap L^{2} \times L^{2} \cap L^{\infty}\right)$. Then

$$
\|u(t)\|_{2} \rightarrow 0, \quad \text { and } \quad\|B(t)\|_{2} \rightarrow M
$$

as $t \rightarrow \infty$, where $M$ is some positive constant.

The previous theorem shows that the diffusion in the velocity is sufficient to prevent compensate oscillations between the two energies.

The next question we addressed is in regards to decay of solutions to MHD equations with diffusion both in the velocity and the magnetic field. Here it is supposed that the data is only in $L^{2}$ space. In this case it is shown that the energies of the velocity and the magnetic field decay to zero without a rate. Moreover, it is 
shown that this is optimal, that is, that cannot be a uniform rate for the energy of the solutions with data exclusively in $L^{2}$. We show that

Theorem 2. Let $n=2,3$. If $(u, B)$ is a weak solution to the MHD equations with $\delta>0$ and data $\left(u_{0}, B_{0}\right) \in\left(L^{2}\left(\mathbb{R}^{n}\right)\right)^{2}$, then

$$
\lim _{t \rightarrow \infty}\left(\|u(t)\|_{2}^{2}+\|B(t)\|_{2}^{2}\right)=0 .
$$

The proof of this theorem is given first formally. To make the result rigorous we apply the method to smooth approximations and then pass to the limit.

With regard to the optimality of this last result we show that

Theorem 3. There exist no functions $G(t, \beta)$ and $H(t, \gamma)$ with the following two properties. If $(u, B)$ is a solution to equations (1.9)-1.5) with $\delta>0$ and data $\left(u_{0}, B_{0}\right) \in\left(L^{2}\left(\mathbb{R}^{n}\right)\right)^{2}, n=2,3$, then

i) $\|u(t)\|_{2} \leq G\left(t,\left\|u_{0}\right\|_{2}\right)$,

ii) $\lim _{t \rightarrow 0} G(t, \beta)=0$, for all $\beta>0$.

The last part of the paper focuses on extending Kato's pioneering work on $L^{p}$ decay for Navier-Stokes equations Kat84 to the MHD equations with magnetic diffusion. In particular we note that a simple modification of Kato's work Kat84 yields equivalent results for the MHD equations, then combined with our result on non-uniform decay in $L^{2}$ gives a slight improvement of the decay rates.

Corollary 4. There is $\lambda>0$ such that for $\left\|u_{0}\right\|_{2} \leq \lambda$ the global solution of the equation (6.1) for $q \geq m$, and for $2 \leq r \leq q$

$$
\lim _{t \rightarrow \infty} t^{\frac{r-2}{2 r}}\|(u, B)\|_{r}=0
$$

\section{Notation}

We denote

$$
\begin{aligned}
& C_{0, \sigma}^{\infty}=C_{0, \sigma}^{\infty}\left(\mathbb{R}^{n}\right): \text { space of infinitely differentiable functions with compact } \\
& \text { support and divergence free, } \\
& L_{\sigma}^{2}=L_{\sigma}^{2}\left(\mathbb{R}^{n}\right): \text { completion of } C_{0, \sigma}^{\infty} \text { in the } L^{2} \text {-norm }\|\cdot\|_{2}, \\
& \dot{H}_{\sigma}^{1}=\dot{H}_{\sigma}^{1}\left(\mathbb{R}^{n}\right): \text { completion of } C_{0, \sigma}^{\infty} \text { in the homogeneous } H^{1} \text {-norm }\|\nabla \cdot\|_{2} .
\end{aligned}
$$

The Fourier transform of $\varphi$ will be denoted by $\mathscr{F}\{\varphi\}=\hat{\varphi}=\int_{\mathbb{R}^{n}} e^{-i \xi x} \varphi(x) d x$ and its inverse transform by $\mathscr{F}^{-1}\{\varphi\}=\check{\varphi}=\frac{1}{2 \pi^{n}} \int e^{i x \xi} \hat{\varphi}(\xi) d \xi$. Also,

$L^{p}\left(a, b ; L^{q}\right)=\left\{f:(a, b) \times \mathbb{R}^{n} \rightarrow \mathbb{R}^{n}:\|f\|_{L^{p}\left(a, b ; L^{q}\right)}=\left(\int_{a}^{b}\|f(\tau)\|_{q}^{p} d \tau\right)^{1 / p}<\infty\right\}$.

The notation $\|\cdot\|_{L^{p, q}}$ will be used for the norm of $L^{p}\left(0, \infty ; L^{q}\right)$, and $\langle f, g\rangle=\int f g d x$ for the inner product in $L^{2}$. Various constants are simply denoted by $C$. 


\section{Preliminary Results}

In this section auxiliary results which will be needed in the sequel are obtained. We consider the MHD equations with no magnetic diffusion $(\delta=0)$. Some of the results presented are standard and their proofs are included in the appendix for completeness. The results below follow ideas of ORS97.

We start with an estimate for the Fourier transform of the velocity.

Proposition 1. Let $(u, B)$ be a mild solution to the MHD equations (1.8)-(1.5). Assume the initial data $u_{0}, B_{0}$ is in $L^{1}\left(\mathbb{R}^{3}\right) \cap L^{2}\left(\mathbb{R}^{3}\right)$. Then

$$
|\hat{u}(t)| \leq C\left(1+\frac{1}{|\xi|}\right)
$$

where $C$ is a constant.

Proof. See Appendix, Proposition 3.

The proofs in this section are formal. To make them rigorous is suffices to apply them to approximations using retarded mollifications such as the ones constructed by [CKN82, Ler34, and HX05b] for the MHD equations and then pass to the limit. For details regarding our proofs see ORS97 were the procedure has been done for the solutions to the Navier-Stokes equations. The extension to MHD is straightforward.

We first recall a standard energy inequality

$$
\|u(t)\|_{2}^{2}+\|B(t)\|_{2}^{2}+2 \int_{0}^{t}\|\nabla u(s)\|_{2}^{2} d s=\left\|u_{0}\right\|_{2}^{2}+\left\|B_{0}\right\|_{2}^{2} .
$$

This follows easily by multiplying (1.2) by $u$, (1.3) by $B$, adding the equations, and then integrating in space and time.

The next proposition gives a generalized energy inequality.

Proposition 2. Assume $(u, B)$ is a weak solution of Equations (1.9)-1.5). For $E(t) \in C^{1}\left(\mathbb{R} ; \mathbb{R}_{+}\right)$with $E(t) \geq 0$ and $\psi \in C^{1}\left(\mathbb{R} ; C^{1} \cap L^{2}\right)$, the weak solution satisfies

$$
\begin{aligned}
E(t)\|\psi * u(t)\|_{2}^{2} & =E(s)\|\psi * u(s)\|_{2}^{2}+\int_{s}^{t} E^{\prime}(\tau)\|\psi * u(\tau)\|_{2}^{2} d \tau \\
& +2 \int_{s}^{t} E(\tau)\left(\left\langle\psi^{\prime} * u(\tau), \psi * u(\tau)\right\rangle-\|\nabla \psi * u(\tau)\|_{2}^{2}\right) d \tau \\
& -2 \int_{s}^{t} E(\tau)(\langle u \cdot \nabla u(\tau), \psi * \psi * u(\tau)\rangle \\
& +\langle B \cdot \nabla B(\tau), \psi * \psi * u(\tau)\rangle) d \tau
\end{aligned}
$$

Proof. Multiply Equation (1.2) by $E(t) \psi * \psi * u(t)$ and integrate by parts to get

$$
\begin{aligned}
\frac{d}{d t}\left(E(t)\|\psi * u(t)\|_{2}^{2}\right)= & E^{\prime}(t)\|\psi * u(t)\|^{2}+2 E(t)\left\{\left\langle\psi^{\prime} * u(t), \psi * u(t)\right\rangle-\|\nabla \psi * u(t)\|_{2}^{2}\right\} \\
& -2 E(t)\{\langle u \cdot \nabla u, \psi * \psi * u(t)\rangle-\langle B \cdot \nabla B, \psi * \psi * u(t)\rangle\}
\end{aligned}
$$

Integrating the preceding equation in the interval $(s, t)$ yields Equation (3.1).

Corollaries a and 6 follow as an easy consequence. 
Corollary 5. Let $(u, B)$ be a weak solution of 1.9)-1.5). Let $\varphi \in L^{2}\left(\mathbb{R}^{3}\right)$, then

$$
\begin{aligned}
\|\check{\varphi} * u(t)\|_{2}^{2} \leq\left\|e^{\Delta(t-s)} \check{\varphi} * u(s)\right\|_{2}^{2}+ & 2 \int_{s}^{t}\left(\left|\left\langle u \cdot \nabla u, e^{2 \Delta(t-\tau)} \breve{\varphi}^{2} * u(\tau)\right\rangle\right|\right. \\
& \left.+\left|\left\langle B \cdot \nabla B, e^{2 \Delta(t-\tau)} \breve{\varphi}^{2} * u(\tau)\right\rangle\right|\right) d \tau .
\end{aligned}
$$

Proof. Choose $E(t)=1$ and $\psi(\tau)$ as

$$
\psi(\tau)=\mathscr{F}^{-1}\left\{e^{-|\xi|^{2}(t+\eta-\tau)} \varphi(-\xi)\right\}, \quad \eta>0
$$

in Eq. (3.1). Then $\psi(\tau) * u(\tau)=e^{\Delta(t+\eta-\tau)} \check{\varphi} * u(\tau)=\int e^{\Delta(t+\eta-\tau+\delta)} \check{\varphi}(x) u(\delta) d \delta$, and

$$
\begin{aligned}
\left\langle\psi^{\prime}(\tau)\right. & * u(\tau), \psi(\tau) * u(\tau)\rangle-\|\nabla \psi(\tau) * u(\tau)\|_{2}^{2} \\
& =-\left\langle\Delta\left(e^{\Delta(t+\eta-\tau)} \check{\varphi}\right) * u(\tau), e^{\Delta(t+\eta-\tau)} \check{\varphi} * u(\tau)\right\rangle-\left\|\nabla\left(e^{\Delta(t+\eta-\tau)} \check{\varphi}\right) * u(\tau)\right\|_{2}^{2} \\
& =0 .
\end{aligned}
$$

Hence we have from (3.1)

$$
\begin{aligned}
\left\|e^{\Delta \eta} \check{\varphi} * u(t)\right\|_{2}^{2} \leq & \left\|e^{\Delta(t+\eta-s)} \breve{\varphi} * u(s)\right\|_{2}^{2} \\
& +2 \int_{s}^{t}\left(\left|\left\langle u \cdot \nabla u, e^{2 \Delta(t+\eta-\tau)} \breve{\varphi}^{2} * u(\tau)\right\rangle\right|\right. \\
& \left.+\left|\left\langle B \cdot \nabla B, e^{2 \Delta(t+\eta-\tau)} \check{\varphi}^{2} * u(\tau)\right\rangle\right|\right) d \tau
\end{aligned}
$$

Let $\eta \rightarrow 0$ in the preceding equation to obtain (3.2).

Corollary 6. Let $E(t) \in C^{1}\left(\mathbb{R}^{+} ; \mathbb{R}\right)$ and $\tilde{\psi} \in C^{1}\left(0, \infty ; L^{\infty} \cap L^{2}\right)$. Then a weak solution of Equations (1.2)-(1.5) satisfies

$$
\begin{aligned}
E(t)\|\tilde{\psi}(t) \hat{u}(t)\|_{2}^{2} \leq & E(s)\|\tilde{\psi}(s) \hat{u}(s)\|_{2}^{2}+\int_{s}^{t} E^{\prime}(\tau)\|\tilde{\psi}(\tau) \hat{u}(\tau)\|_{2}^{2} d \tau \\
& +2 \int_{s}^{t} E(\tau)\left(\left\langle\tilde{\psi}^{\prime}(\tau) \hat{u}(\tau), \bar{\psi}(\tau) \hat{u}(\tau)\right\rangle-\|\xi \tilde{\psi}(\tau) \hat{u}(\tau)\|_{2}^{2}\right) d \tau \\
& -2 \int_{s}^{t} E(\tau)\left(\left\langle\mathscr{F}\{u \cdot \nabla u(\tau)\}, \tilde{\psi}^{2} \hat{u}(\tau)\right\rangle\right. \\
& \left.+\left\langle\mathscr{F}\{B \cdot \nabla B(\tau)\}, \tilde{\psi}^{2} \hat{u}(\tau)\right\rangle\right) d \tau,
\end{aligned}
$$

for almost all $s \geq 0$ and all $t \geq s$.

Proof. Apply Plancherel's theorem to (3.1).

\section{Non-uniform DeCAy of SOlutions to the MHD EQUations With NO} MAGNETIC DIFFUSION

In this section it is shown that if there were to exist strong bounded solutions in $3 \mathrm{D}$, they can not have compensatory oscillations. We analyze separately the energy of the high and low frequencies of the solutions. The main tool for the analysis of the high frequency is Fourier Splitting, see Sch85. 
We first establish Theorem 11 of the introduction, which we recall for completeness.

Theorem 1. Let $n=3$. Suppose that there exists $(u, B)$ a strong bounded solution to the MHD equations with $\delta=0$, magnetic field bounded in $L^{\infty}$, and data $\left(u_{0}, B_{0}\right) \in\left(L^{1} \cap L^{2} \times L^{2} \cap L^{\infty}\right)$. Then

$$
\|u(t)\|_{2} \rightarrow 0, \quad \text { and } \quad\|B(t)\|_{2} \rightarrow M,
$$

as $t \rightarrow \infty$, where $M$ is some positive constant.

Proof. Split the velocity of the solution into low and high frequency parts

$$
\|u(t)\|_{2}=\|\hat{u}(t)\|_{2} \leq\|\varphi \hat{u}\|_{2}+\|(1-\varphi) \hat{u}\|_{2},
$$

where $\varphi$ is a function in Fourier space to be chosen appropriately, to emphasize the low and high frequency of $u$.

Low frequency Decay. Set $\varphi(\xi)=e^{-|\xi|^{2} t}$, using the result of Corollary 5 and Plancherel theorem,

$$
\begin{aligned}
\|\varphi \hat{u}(t)\|^{2} \leq & \left\|e^{-|\xi|^{2}(t-s)} \varphi \hat{u}(s)\right\|^{2}+2 \int_{s}^{t}\left|\left\langle u \cdot \nabla u, e^{2 \Delta(t-\tau)} \check{\varphi}^{2} * u\right\rangle\right| d \tau \\
& +2 \int_{s}^{t}\left|\left\langle B \cdot \nabla B, e^{2 \Delta(t-\tau)} \breve{\varphi}^{2} * u\right\rangle\right| d \tau \\
\leq & \left\|e^{-|\xi|^{2}(t-s)} \varphi \hat{u}(s)\right\|_{2}^{2}+2 \int_{s}^{t}\left|\left\langle\check{\varphi}^{2} * u \cdot \nabla u, e^{2 \Delta(t-\tau)} u\right\rangle\right| d \tau \\
& +2 \int_{s}^{t}\left|\left\langle\check{\varphi}^{2} * B \cdot \nabla B, e^{2 \Delta(t-\tau)} u\right\rangle\right| d \tau
\end{aligned}
$$

Clearly the first term on the right hand side satisfies

$$
\limsup _{t \rightarrow \infty}\left\|e^{-|\xi|^{2}(t-s)} \varphi \hat{u}(s)\right\|_{2}^{2}=0 .
$$

To bound the third term note first that

$$
\begin{aligned}
\left\|\breve{\varphi}^{2} * B \cdot \nabla B\right\|_{2}^{2} & =\sum_{j} \int\left|\breve{\varphi}^{2} *(B \cdot \nabla) B^{j}\right|^{2} d x \leq C \sum_{j}\left(\sum_{i}\left\|\partial_{i} \check{\varphi}^{2} * B^{i} B^{j}\right\|_{2}\right)^{2} \\
& \leq C\|B\|_{2}^{2}\left(\sum_{i}\left\|\partial_{i} \check{\varphi}^{2}\right\|_{2}\right)^{2}
\end{aligned}
$$

where $C$ is a positive constant.

Thus

$$
\left|\left\langle\breve{\varphi}^{2} * B \cdot \nabla B, e^{2 \Delta(t-\tau)} u\right\rangle\right| \leq C\|B(\tau)\|_{2}^{2}\|u(\tau)\|_{2}\left(\sum_{i}\left\|\partial_{i} \check{\varphi}^{2}\right\|_{2}\right)
$$

Since $\check{\varphi}^{2}=(4 \pi t)^{-3} e^{-|x|^{2} / 2 t}$, it follows that

$$
\left\|\partial_{i} \check{\varphi}^{2}\right\|_{2}^{2} \leq C \int_{\mathbb{R}^{3}}\left(t^{-4}|x| e^{-|x|^{2} / 2 t}\right)^{2} d x \leq C t^{-11 / 2} \int_{\mathbb{R}^{3}} \frac{e^{-|x|^{2} / 2 t}}{t^{3 / 2}} d x=C t^{-11 / 2} .
$$


Hence,

$$
\left|\left\langle\breve{\varphi}^{2} * B \cdot \nabla B, e^{2 \Delta(t-\tau)} u\right\rangle\right| \leq C\|B(\tau)\|_{2}\|u(\tau)\|_{2} t^{-11 / 2} \leq C t^{-11 / 2},
$$

since the $L^{2}$-norm of $u$ and $B$ are bounded by the initial data.

Similarly, $\left|\left\langle\breve{\varphi}^{2} * u \cdot \nabla u, e^{2 \Delta(t-\tau)} u\right\rangle\right| \leq C t^{-11 / 2}$. Hence by (4.1), (4.2), and the last two inequalities

$$
\|\varphi \check{u}(t)\|_{2}^{2} \rightarrow 0 \quad \text { as } \quad t \rightarrow \infty .
$$

High frequency Decay. We will show that

$$
\lim _{t \rightarrow \infty}\|(1-\varphi) u\|_{2} \leq \varepsilon_{0}
$$

for all $\varepsilon_{0}>0$. The Fourier splitting method will be used. Let $\chi(\varepsilon)=\{\xi:|\xi| \leq$ $G(\varepsilon)\}$, a neighborhood of the origin, were $G$ will be specified below. Set $\tilde{\psi}=1-\varphi$, where $\varphi$ is given above. Then $\tilde{\psi}^{\prime}=|\xi|^{2} \varphi$, and Corollary (6) yields

$$
\begin{aligned}
E(t)\|(1-\varphi) \hat{u}(t)\|_{2}^{2} & \leq E(s)\|(1-\varphi) \hat{u}(s)\|_{2}^{2}+\int_{s}^{t} E^{\prime}(\tau) \int_{\chi(\varepsilon)}|(1-\varphi) \hat{u}(\tau)|^{2} d \xi d \tau \\
& +\int_{s}^{t} E^{\prime}(\tau) \int_{R^{3} \backslash \chi(\varepsilon)}|(1-\varphi) \hat{u}(\tau)|^{2} d \xi d \tau \\
& -2 \int_{s}^{t} E(\tau)\|\xi(1-\varphi) \hat{u}(\tau)\|_{2}^{2} d \tau \\
& +2 \int_{s}^{t} E(\tau)\left\langle|\xi|^{2} \varphi(\tau) \hat{u}(\tau),(1-\varphi(\tau)) \hat{u}(\tau)\right\rangle d \tau \\
& +2 \int_{s}^{t} E(\tau)\left|\left\langle\widehat{u \cdot \nabla u},(1-\varphi)^{2} \hat{u}(\tau)\right\rangle\right| d \tau \\
& +2 \int_{s}^{t} E(\tau)\left|\left\langle\widehat{B \cdot \nabla B},(1-\varphi)^{2} \hat{u}(\tau)\right\rangle\right| d \tau .
\end{aligned}
$$

The terms in the second and third row are bounded by

$$
\int_{s}^{t}\left(E^{\prime}(\tau)-2 E(\tau) G^{2}(\varepsilon)\right) \int_{\mathbb{R}^{3} \backslash \chi(\varepsilon)}|(1-\varphi) \hat{u}(\tau)|^{2} d \xi d \tau .
$$

Choose $E(t)=e^{\varepsilon t}$ and $G(\varepsilon)=\sqrt{\varepsilon / 2}$ hence $E^{\prime}(t)-2 E(t) G^{2}(\varepsilon)=0$, thus the above integral vanishes.

Divide Equation (4.4) by $E(t)$,

$$
\begin{aligned}
& \|(1-\varphi) \hat{u}(t)\|_{2}^{2} \leq \frac{E(s)}{E(t)}\|(1-\varphi) \hat{u}(s)\|_{2}^{2}+\frac{1}{E(t)} \int_{s}^{t} E^{\prime}(\tau) \int_{\chi(\varepsilon)}|(1-\varphi) \hat{u}(\tau)|^{2} d \xi d \tau \\
& +\frac{2}{E(t)} \int_{s}^{t} E(\tau)\left\langle|\xi|^{2} \varphi(\tau) \hat{u}(\tau),(1-\varphi(\tau)) \hat{u}(\tau)\right\rangle d \tau \\
& +\frac{2}{E(t)} \int_{s}^{t} E(\tau)\left|\left\langle\widehat{u \cdot \nabla u},(1-\varphi)^{2} \hat{u}(\tau)\right\rangle\right| d \tau+\frac{2}{E(t)} \int_{s}^{t} E(\tau)\left|\left\langle\widehat{B \cdot \nabla B},(1-\varphi)^{2} \hat{u}(\tau)\right\rangle\right| d \tau \\
& =I(t)+I I(t)+I I I(t)+I V(t)+V(t) .
\end{aligned}
$$


ESTIMATE FOR $I(t)$ :

$$
I(t)=\frac{E(s)}{E(t)}\|(1-\varphi) \hat{u}(s)\|_{2}^{2} \leq e^{\varepsilon(s-t)}\|\hat{u}(s)\|_{2}^{2} \leq\left(\left\|u_{0}\right\|_{2}^{2}+\left\|B_{0}\right\|_{2}^{2}\right) e^{\varepsilon(s-t)} \leq C e^{\varepsilon(s-t)} .
$$

Hence

$$
\lim _{t \rightarrow \infty} I(t)=\lim _{t \rightarrow \infty} \frac{E(s)}{E(t)}\|(1-\varphi) \hat{u}(s)\|_{2}^{2}=0 .
$$

Estimate FOR $I I(t)$ : By Proposition 1

$$
\int_{\chi(\varepsilon)}|\hat{u}|^{2} d \xi \leq C \int_{\chi(\varepsilon)}\left(1+\frac{1}{|\xi|}\right)^{2} d \xi \leq C \int_{\chi(\varepsilon)}\left(1+\frac{1}{|\xi|^{2}}\right) d \xi \leq C\left(\varepsilon^{3 / 2}+\varepsilon^{1 / 2}\right) .
$$

Since $(1-\varphi)^{2} \leq 1$

$$
\begin{aligned}
I I(t) & =\frac{1}{E(t)} \int_{s}^{t} E^{\prime}(\tau) \int_{\chi(\varepsilon)}|(1-\varphi) \hat{u}(\tau)|^{2} d \xi d \tau \\
& \leq \frac{1}{E(t)} \int_{s}^{t} E^{\prime}(\tau) \int_{\chi(\varepsilon)}|\hat{u}(\tau)|^{2} d \xi d \tau \leq C \varepsilon^{1 / 2}=\varepsilon_{0} .
\end{aligned}
$$

Estimate FOR $I I I(t)$ : Observe that $0 \leq \varphi-\varphi^{2} \leq 1$, and $E(\tau) \leq E(t)$ for $\tau<t$, hence

$$
\begin{aligned}
I I I(t) & =\frac{2}{E(t)} \int_{s}^{t} E(\tau)\left\langle|\xi|^{2} \varphi(\tau) \hat{u}(\tau),(1-\varphi(\tau)) \hat{u}(\tau)\right\rangle d \tau \\
& \leq \frac{2}{E(t)} \int_{s}^{t} E(t) \int_{\mathbb{R}^{3}}|\xi|^{2}\left(\varphi-\varphi^{2}\right)|\hat{u}(\tau)|^{2} d \xi d \tau \leq C \int_{s}^{t}\|\nabla u(\tau)\|_{2}^{2} d \tau,
\end{aligned}
$$

Since $\int_{0}^{\infty}\|\nabla u\|_{2}^{2}<\infty$ it follows that

$$
\lim _{t \rightarrow \infty} I I I(t) \leq C \lim _{s \rightarrow \infty} \lim _{t \rightarrow \infty} \int_{s}^{t}\|\nabla u(\tau)\|_{2}^{2} d \tau=0
$$

Estimate FOR $I V(t)$ : Set $\zeta=\mathscr{F}^{-1}\left\{1-(1-\varphi)^{2}\right\}$. This function is essentially the heat kernel. Note that $\langle u \cdot \nabla u, u\rangle=0$, hence $I V(t)$ can be estimated as follows

$$
\begin{aligned}
I V(t) & =\frac{2}{E(t)} \int_{s}^{t} E(\tau)|\langle u \cdot \nabla u, \zeta * u\rangle| d \tau \leq C \int_{s}^{t}\|\zeta\|_{6 / 5}\|u \cdot \nabla u\|_{3 / 2}\|u\|_{2} d \tau \\
& \leq C \int_{s}^{t}\|\zeta\|_{6 / 5}\|u\|_{6}\|\nabla u\|_{2} d \tau \leq C \int_{s}^{t}\|\zeta\|_{6 / 5}\|\nabla u\|_{2}^{2} d \tau \\
& \leq C \int_{s}^{t} \frac{\|\nabla u\|_{2}^{2}}{\tau^{1 / 4}} d \tau \leq \frac{C}{s^{1 / 4}} \int_{s}^{t}\|\nabla u\|_{2}^{2} d \tau .
\end{aligned}
$$

Thus

$$
\lim _{t \rightarrow \infty} I V(t) \leq \lim _{s \rightarrow \infty} \lim _{t \rightarrow \infty} \frac{C}{s^{1 / 4}} \int_{s}^{t}\|\nabla u\|_{2}^{2} d \tau=0 .
$$

ESTIMATE FOR $V(t)$ : 


$$
\begin{aligned}
V(t) & =\frac{2}{E(t)} \int_{s}^{t} E(\tau)\left|\left\langle\widehat{B \cdot \nabla B},(1-\varphi)^{2} \hat{u}(\tau)\right\rangle\right| d \tau \leq \frac{C}{E(t)} \int_{s}^{t} E(\tau)\left(\sum_{j} \int|\widehat{B \cdot B}|\left|\widehat{\nabla u^{j}}\right| d \xi\right) d \tau \\
& \leq \frac{C}{E(t)} \int_{s}^{t} E(\tau)\left(\int|B \cdot B|^{2} d \xi\right)^{1 / 2}\left(\int|\nabla u(\tau)|^{2} d \xi\right)^{1 / 2} d \tau \\
& \leq \frac{C}{E(t)} \int_{s}^{t} E(\tau)\left(\int|B(\tau)|^{4} d \xi\right)^{1 / 2}\|\nabla u(\tau)\|_{2} d \tau \\
& \leq \frac{C}{E(t)} \int_{s}^{t} E(\tau)\|B\|_{\infty}\|B(\tau)\|_{2}\|\nabla u(\tau)\|_{2} d \tau \\
& \leq \frac{C}{E(t)} \int_{s}^{t} E(\tau)\|\nabla u(\tau)\|_{2} d \tau,
\end{aligned}
$$

Here is the only place where we need that the magnetic field is bounded. Specifically we use that $B \in L^{\infty}\left(\mathbb{R}^{3} \times \mathbb{R}_{+}\right)$. Since $B \in L^{2}$ we get the above bound. Recall that $E(t)=e^{\varepsilon t}$, hence

$$
\begin{aligned}
V(t) & \leq \frac{C}{E(\tau)} \int_{s}^{t} E(t)\|\nabla u(\tau)\|_{2} d \tau \leq \frac{C}{e^{\varepsilon t}}\left(\frac{e^{2 \varepsilon t}}{2 \varepsilon}\right)^{1 / 2}\left(\int_{s}^{t}\|\nabla u(\tau)\|_{2}^{2} d \tau\right)^{1 / 2} \\
& =\frac{C}{\sqrt{2 \varepsilon}}\left(\int_{s}^{t}\|\nabla u(\tau)\|_{2}^{2} d \tau\right)^{1 / 2},
\end{aligned}
$$

which as before tends to zero as $t$ and $s$ goes to infinity.

Combining the estimates $I(t)-V(t)$ yields In summary, we have showed that

$$
\lim _{t \rightarrow \infty}\|(1-\varphi) \hat{u}(t)\|_{2} \leq \varepsilon_{0} .
$$

Since $\varepsilon_{0}$ is arbitrary and positive, combining (4.1) and (4.6) yields

$$
\lim _{t \rightarrow \infty}\|u(t)\|_{2}=0 \text {. }
$$

To obtain the limit of $\|B\|_{2}$ proceed as follows. Set

$$
\phi(t)=\|u(t)\|_{2}^{2}+\|B(t)\|_{2}^{2} .
$$

Given that $\phi(t) \geq 0$ and is decreasing, there exists a constant $M$ such that $\phi(t) \rightarrow$ $M$ as $t \rightarrow \infty$. Since $\|u(t)\|_{2} \rightarrow 0$, it follows that

$$
\|B(t)\|_{2} \rightarrow M \quad \text { as } t \rightarrow \infty .
$$

This completes the proof.

\section{MHD EQUATIONS With DIFFUSION}

In this section it is shown that if the data is only in $L^{2}$ then the solution decays without a rate. The ideas of the proof are similar to Theorem 1 i only that due to the added magnetic diffusion we need less information on the data. The main result

of this section is that this decay is optimal. Specifically it is shown that the decay can not be uniform. 
The proof we give below is formal. To make it rigorous, it can be applied to smooth approximations and then pass to the limit. The approximations could be constructed by retarded mollification as was done for the Navier-Stokes equations in CKN82, Ler34. This construction if modified for the MHD equations will give suitable approximations which can be used to make our arguments rigorous. This arguments are standard and as such will be omitted. To see the construction of these approximations in detail we refer the reader to HX05b.

\subsection{Non-uniform decay.}

Theorem 2. Let $n=2,3$. If $(u, B)$ is a weak solution to the MHD equations with $\delta>0$, and data $\left(u_{0}, B_{0}\right) \in\left(L^{2}\left(\mathbb{R}^{n}\right)\right)^{2}$, then

$$
\lim _{t \rightarrow \infty}\left(\|u(t)\|_{2}^{2}+\|B(t)\|_{2}^{2}\right)=0
$$

Proof. Without loss of generality suppose $\delta=1$. The proof is based on similar arguments given in [ORS97 for solutions to the Navier-Stokes equations with a forcing term.

Let $\phi(\xi)=e^{-|\xi|^{2}}$. As before split $u$ into low and high frequency parts

$$
\begin{aligned}
\|u(t)\|_{2} & =\|\hat{u}(t)\|_{2} \leq\|\varphi \hat{u}\|_{2}+\|(1-\varphi) \hat{u}\|_{2}, \\
\|B(t)\|_{2} & =\|\hat{B}(t)\|_{2} \leq\|\varphi \hat{B}\|_{2}+\|(1-\varphi) \hat{B}\|_{2} .
\end{aligned}
$$

Low frequency decay. We need to use Corollary (5) and Plancherel's identity.

$$
\begin{aligned}
\|\varphi \hat{u}(t)\|^{2} \leq & \left\|e^{-|\xi|^{2}(t-s)} \varphi \hat{u}(s)\right\|_{2}^{2}+2 \int_{s}^{t}\left|\left\langle\breve{\varphi}^{2} * u \cdot \nabla u, e^{2 \Delta(t-\tau)} u\right\rangle\right| d \tau \\
& +2 \int_{s}^{t}\left|\left\langle\check{\varphi}^{2} * B \cdot \nabla B, e^{2 \Delta(t-\tau)} u\right\rangle\right| d \tau=I_{1}+I_{2}+I_{3},
\end{aligned}
$$

and

$$
\begin{aligned}
\|\varphi \hat{B}(t)\|^{2} \leq & \left\|e^{-|\xi|^{2}(t-s)} \varphi \hat{B}(s)\right\|_{2}^{2}+2 \int_{s}^{t}\left|\left\langle\breve{\varphi}^{2} * B \cdot \nabla B, e^{2 \Delta(t-\tau)} u\right\rangle\right| d \tau \\
& +2 \int_{s}^{t}\left|\left\langle\check{\varphi}^{2} * u \cdot \nabla B, e^{2 \Delta(t-\tau)} u\right\rangle\right| d \tau=J_{1}+J_{2}+J_{3} .
\end{aligned}
$$

It is immediate that the first terms $I_{1}$ and $J_{1}$ in (5.1), (5.2) tend to zero respectively as $t$ goes to infinity. Hence it will be only necessary to show that the two integrals on the right hand side of each of the above equations tends to zero when $t$ goes to infinity. Since all integrals can be estimated in a similar fashion we will only analyze the integrals $I_{2}$, and $I_{3}$ corresponding to the velocity.

Since $\breve{\varphi}^{2}$ is a rapidly decreasing function, by the Hasdorff-Young, Hölder, and Sobolev inequalities we have the following.

When $n=2$

$$
\begin{aligned}
\left|\left\langle\check{\varphi}^{2} * u \cdot \nabla u, e^{2 \Delta(t-\tau)} u\right\rangle\right| & \leq\left|\left\langle u \cdot \nabla \check{\varphi}^{2} * e^{2 \Delta(t-\tau)} u, u\right\rangle\right| \leq\|u\|_{4}^{2}\left\|\check{\varphi}^{2} * e^{2 \Delta(t-\tau)} \nabla u\right\|_{2} \\
& \leq\left\|\check{\varphi}^{2}\right\|_{\infty}\|u\|_{4}^{2}\|\nabla u\|_{2} \leq C\|u\|_{2}\|\nabla u\|_{2}^{2}
\end{aligned}
$$


and

$$
\begin{aligned}
\left|\left\langle\check{\varphi}^{2} * B \cdot \nabla B, e^{2 \Delta(t-\tau)} u\right\rangle\right| & \leq\left|\left\langle B \cdot \nabla \check{\varphi}^{2} * e^{2 \Delta(t-\tau)} B, u\right\rangle\right| \leq\|B\|_{4}^{2}\left\|\check{\varphi}^{2} * e^{2 \Delta(t-\tau)} \nabla u\right\|_{2} \\
& \leq\left\|\check{\varphi}^{2}\right\|_{\infty}\|B\|_{4}^{2}\|\nabla u\|_{2} \leq C\|B\|_{2}\|\nabla B\|_{2}\|\nabla u\|_{2} \\
& \leq C\left(\|\nabla B\|_{2}^{2}+\|\nabla u\|_{2}^{2}\right) .
\end{aligned}
$$

For $n=3$

$$
\begin{aligned}
\left|\left\langle\check{\varphi}^{2} * u \cdot \nabla u, e^{2 \Delta(t-\tau)} u\right\rangle\right| & \leq\left\|\check{\varphi}^{2} * u \cdot \nabla u\right\|_{2}\|u\|_{2} \leq C\left\|\check{\varphi}^{2}\right\|_{6 / 5}\|u \cdot \nabla u\|_{3 / 2}\|u\|_{2} \\
& \leq C\|u\|_{6}\|\nabla u\|_{2} \leq C\|u\|_{2}\|\nabla u\|_{2}^{2} .
\end{aligned}
$$

In the same fashion

$$
\begin{aligned}
\left|\left\langle\check{\varphi}^{2} * B \cdot \nabla B, e^{2 \Delta(t-\tau)} u\right\rangle\right| & \leq\left\|\check{\varphi}^{2} * B \cdot \nabla B\right\|_{2}\|u\|_{2} \leq C\left\|\check{\varphi}^{2}\right\|_{6 / 5}\|B \cdot \nabla B\|_{3 / 2}\|u\|_{2} \\
& \leq C\|B\|_{6}\|\nabla u\|_{2} \leq C\|u\|_{2}\|\nabla B\|_{2}^{2} .
\end{aligned}
$$

Hence integrating over $(s, t)$ yields

$$
I_{2}+I_{3} \leq C \int_{s}^{t}\|\nabla B\|_{2}^{2}+\|\nabla u\|_{2}^{2} d \tau
$$

Thus

$$
\lim _{t \rightarrow \infty} I_{2}+I_{3} \leq \lim _{s \rightarrow \infty} \lim _{t \rightarrow \infty} C \int_{s}^{t}\|\nabla B\|_{2}^{2}+\|\nabla u\|_{2}^{2} d \tau=0 .
$$

In the same manner it follows that the $\lim _{t \rightarrow \infty} J_{2}+J_{3}=0$. Hence

$$
\lim _{t \rightarrow \infty}\|\varphi \hat{u}\|_{2}=0, \quad \lim _{t \rightarrow \infty}\|\varphi \hat{B}\|_{2}=0 .
$$

High frequency decay. To estimate the high frequency part we will use Fourier Splitting Sch85. We now use Corollary (5) and an equivalent version of this corollary for the magnetic field. Choose $\tilde{\psi}=1-e^{-|\xi|^{2}}=1-\varphi$ (note that in this case $\tilde{\psi}$ is independent of time). Let $\chi(t)=\left\{\xi \in \mathbb{R}^{n}:|\xi| \leq G(t)\right\}$, then

$$
\begin{aligned}
E(t) & {\left[\|(1-\varphi) \hat{u}(t)\|_{2}^{2}+\|(1-\varphi) \hat{B}(t)\|_{2}^{2}\right] \leq E(s)\left(\|(1-\varphi) \hat{u}(s)\|_{2}^{2}+\|(1-\varphi) \hat{B}(s)\|_{2}^{2}\right) } \\
& +\int_{s}^{t} E^{\prime}(\tau) \int_{\chi(t)}|(1-\varphi) \hat{u}(\tau)|^{2} d \xi d \tau+\int_{s}^{t} E^{\prime}(\tau) \int_{\chi(t)}|(1-\varphi) \hat{B}(\tau)|^{2} d \xi d \tau \\
& +\int_{s}^{t} E^{\prime}(\tau) \int_{R^{3} \backslash \chi(t)}|(1-\varphi) \hat{u}(\tau)|^{2} d \xi d \tau+\int_{s}^{t} E^{\prime}(\tau) \int_{R^{3} \backslash \chi(t)}|(1-\varphi) \hat{B}(\tau)|^{2} d \xi d \tau \\
& -2 \int_{s}^{t} E(\tau)\|\xi(1-\varphi) \hat{u}(\tau)\|_{2}^{2} d \tau-2 \int_{s}^{t} E(\tau)\|\xi(1-\varphi) \hat{B}(\tau)\|_{2}^{2} d \tau \\
& -2 \int_{s}^{t} E(\tau)\left\langle\widehat{u \cdot \nabla u},(1-\varphi)^{2} \hat{u}(\tau)\right\rangle d \tau+2 \int_{s}^{t} E(\tau)\left\langle\widehat{B \cdot \nabla B},(1-\varphi)^{2} \hat{u}(\tau)\right\rangle d \tau \\
& -2 \int_{s}^{t} E(\tau)\left\langle\widehat{B \cdot \nabla u},(1-\varphi)^{2} \hat{B}(\tau)\right\rangle d \tau+2 \int_{s}^{t} E(\tau)\left\langle\widehat{u \cdot \nabla B},(1-\varphi)^{2} \hat{B}(\tau)\right\rangle d \tau .
\end{aligned}
$$

Suppose

$$
E(t)=(1+t)^{\alpha}, \quad \text { and } \quad G^{2}(t)=\frac{\alpha}{2(1+t)},
$$


with $\alpha>3$. With this choice $E^{\prime}(t)-2 E(t) G^{2}(t)=0$, proceeding as in Theorem 1 will yield

$$
\begin{aligned}
& \int_{s}^{t} E^{\prime}(\tau) \int_{R^{3} \backslash \chi(t)}|(1-\varphi) \hat{u}(\tau)|^{2} d \xi d \tau-2 \int_{s}^{t} E(\tau)\|\xi(1-\varphi) \hat{u}(\tau)\|_{2}^{2} d \tau \\
& \quad+\int_{s}^{t} E^{\prime}(\tau) \int_{R^{3} \backslash \chi(t)}|(1-\varphi) \hat{B}(\tau)|^{2} d \xi d \tau-2 \int_{s}^{t} E(\tau)\|\xi(1-\varphi) \hat{B}(\tau)\|_{2}^{2} d \tau \leq 0 .
\end{aligned}
$$

By (5.6), Equation (5.4) can be reduced to

$$
\begin{aligned}
E(t) & {\left[\|(1-\varphi) \hat{u}(t)\|_{2}^{2}+\|(1-\varphi) \hat{B}(t)\|_{2}^{2}\right] \leq E(s)\left(\|(1-\varphi) \hat{u}(s)\|_{2}^{2}+\|(1-\varphi) \hat{B}(s)\|_{2}^{2}\right) } \\
& +\int_{s}^{t} E^{\prime}(\tau) \int_{\chi(t)}|(1-\varphi) \hat{u}(\tau)|^{2} d \xi d \tau+\int_{s}^{t} E^{\prime}(\tau) \int_{\chi(t)}|(1-\varphi) \hat{B}(\tau)|^{2} d \xi d \tau \\
& -2 \int_{s}^{t} E(\tau)\left\langle\widehat{u \cdot \nabla u},(1-\varphi)^{2} \hat{u}(\tau)\right\rangle d \tau+2 \int_{s}^{t} E(\tau)\left\langle\widehat{B \cdot \nabla B},(1-\varphi)^{2} \hat{u}(\tau)\right\rangle d \tau \\
& -2 \int_{s}^{t} E(\tau)\left\langle\widehat{B \cdot \nabla u},(1-\varphi)^{2} \hat{B}(\tau)\right\rangle d \tau+2 \int_{s}^{t} E(\tau)\left\langle\widehat{u \cdot \nabla B},(1-\varphi)^{2} \hat{B}(\tau)\right\rangle d \tau .
\end{aligned}
$$

We now will bound the terms in (5.8). Observing that $|1-\varphi| \leq|\xi|^{2}$ if $|\xi|<1$, we have

$$
\begin{aligned}
\int_{\chi(\tau)}|(1-\varphi)|^{2}\left(|\hat{u}|^{2}+|\hat{B}|^{2}\right) d \xi & \leq C G(\tau)^{4} \int_{\chi(\tau)}\left(|\hat{u}|^{2}+|\hat{B}|^{2}\right) d \xi \\
& \leq C\left(\left\|u_{0}\right\|_{2}^{2}+\left\|B_{0}\right\|_{2}^{2}\right)(1+\tau)^{-2}
\end{aligned}
$$

We now analyze (5.9), (5.10) together. For this note first that $(1-\varphi)^{2}=1+\theta$, where $\theta=-2 \varphi+\varphi^{2}$, hence by the definition of $\varphi$, the function $\theta$ is a rapidly decreasing function.

Since $\langle u \cdot \nabla u, u\rangle=\langle u \cdot \nabla B, B\rangle=0$, and $\langle\widehat{B \cdot \nabla B}, \hat{u}\rangle-\langle\widehat{u \cdot \nabla B}, \hat{B}\rangle=0$, it follows that the four last terms of the right hand side of (5.4) can be expressed as

$$
\begin{aligned}
-2 \int_{s}^{t} E(\tau)\langle\widehat{u \cdot \nabla u}, \theta \hat{u}(\tau)\rangle d \tau+2 \int_{s}^{t} E(\tau)\langle\widehat{B \cdot \nabla B}, \theta \hat{u}(\tau)\rangle d \tau \\
\quad-2 \int_{s}^{t} E(\tau)\langle\widehat{B \cdot \nabla u}, \theta \hat{B}(\tau)\rangle d \tau+2 \int_{s}^{t} E(\tau)\langle\widehat{u \cdot \nabla B}, \theta \hat{B}(\tau)\rangle d \tau=K_{1}+K_{2}+K_{3}+K_{4} .
\end{aligned}
$$

The estimates of $K_{i}$ 's are all very similar. Hence we only estimate $K_{1}$ and state estimates are for $K_{i}$ for $i=2,3,4$. 
For $n=2$

$$
\begin{aligned}
K_{1} & =\int_{s}^{t} E(\tau)|u \cdot \nabla u, \check{\theta} * u(\tau)| d \tau=\int_{s}^{t} E(\tau)|\langle u \cdot \check{\theta} * \nabla u(\tau), u(\tau)\rangle| d \tau \\
& =\int_{s}^{t} E(\tau)\|\check{\theta}\|_{1}\|u\|_{4}^{2}\|\nabla u\|_{2} d \tau \leq C \int_{s}^{t} E(\tau)\|u\|_{2}\|\nabla u\|_{2}^{2} d \tau \\
& \leq C\left(\left\|u_{0}\right\|_{2}^{2}+\left\|B_{0}\right\|_{2}^{2}\right) \int_{s}^{t} E(\tau)\|\nabla u\|_{2}^{2} d \tau,
\end{aligned}
$$

Same type of computations yields that

$$
K_{i} \leq C \int_{s}^{t} E(\tau)\left(\|\nabla u\|_{2}^{2}+\|\nabla B\|_{2}^{2}\right) d \tau, \quad i=2,3,4 .
$$

For $n=3$ we also only estimate $K_{1}$

$$
\begin{aligned}
\int_{s}^{t} E(\tau)|\langle u \cdot \nabla u, \check{\theta} * u(\tau)\rangle| d \tau & \leq \int_{s}^{t} E(\tau)\|\check{\theta}\|_{6 / 5}\|u \cdot \nabla u\|_{3}\|u\|_{2} d \tau \\
& \leq C \int_{s}^{t} E(\tau)\|u\|_{6}\|\nabla u\|_{2} d \tau \leq C \int_{s}^{t} E(\tau)\|\nabla u\|_{2}^{2} d \tau
\end{aligned}
$$

The same estimates yield

$$
K_{i} \leq C \int_{s}^{t} E(\tau)\left(\|\nabla u\|_{2}^{2}+\|\nabla B\|_{2}^{2}\right) d \tau, \quad i=2,3,4 .
$$

Combining the estimates 5.7)-(5.10), and the estimates for the $K_{i}$ 's yields after division by $E(t)$

$$
\begin{aligned}
\|(1-\varphi) \hat{u}(t)\|_{2}^{2} & +\|(1-\varphi) \hat{B}(t)\|_{2}^{2} \leq \frac{E(s)}{E(t)}\|(1-\varphi) \hat{u}(s)\|_{2}^{2} \\
& +\frac{1}{E(t)} \int_{s}^{t} E^{\prime}(\tau) \int_{\chi(\tau)}|1-\varphi|^{2}\left(|\hat{u}|^{2}+|\hat{B}|^{2}\right) d \xi d \tau \\
& +\frac{C}{E(t)} \int_{s}^{t} E(\tau)\left(\|\nabla u(\tau)\|_{2}^{2}+\|\nabla B(\tau)\|_{2}^{2}\right) d \tau
\end{aligned}
$$

Since $1-\varphi \leq 1$, combining the last equation with (5.11), recalling the definition of $E(t)$ and $G(t)$ in (5.5), and since $\alpha>3$ we have

$$
\begin{aligned}
\lim _{t \rightarrow \infty} & \left(\|(1-\varphi) \hat{u}(t)\|_{2}^{2}+\|(1-\varphi) \hat{B}(t)\|_{2}^{2}\right) \leq \lim _{t \rightarrow \infty}\left(\frac{1+s}{1+t}\right)^{\alpha}\left(\left\|u_{0}\right\|_{2}^{2}+\left\|B_{0}\right\|_{2}^{2}\right) \\
& +C\left(\left\|u_{0}\right\|_{2}^{2}+\left\|B_{0}\right\|_{2}^{2}\right) \lim _{t \rightarrow \infty}\left(\frac{1}{(1+t)^{\alpha}} \int_{s}^{t}(1+t)^{\alpha-3}\right) d \tau \\
& +\lim _{t \rightarrow \infty} \frac{C}{E(t)} \int_{s}^{t} E(\tau)\left(\|\nabla u\|_{2}^{2}+\|\nabla B\|_{2}^{2}\right) d \tau \\
& =C \int_{s}^{\infty}\left(\|\nabla u\|_{2}^{2}+\|\nabla B\|_{2}^{2}\right) d \tau
\end{aligned}
$$

Letting $s \rightarrow \infty$ on the right hand side yields

$$
\lim _{t \rightarrow \infty}\left(\|(1-\varphi) \hat{u}(t)\|_{2}^{2}+\|(1-\varphi) \hat{B}(t)\|_{2}^{2}\right)=0
$$


Combining (5.3) with the last limit gives

$$
\lim _{t \rightarrow \infty}\left(\|u\|_{2}^{2}+\|B\|_{2}^{2}\right)=0
$$

As stated in the beginning to make this proof rigorous, the formal proof has to be applied to the approximating solutions described at the beginning of the section, and then pass to the limit. This procedure is standard and as such is omitted. This completes the proof of the theorem.

5.2. Lack of uniformity. In this subsection it is shown that solutions with data $\left(u_{0}, B_{0}\right)$ in $\left(L^{2}\left(\mathbb{R}^{n}\right)\right)^{2}$ cannot be expected to decay at a uniform rate, in the sense that for each sphere in $L^{2}\left(\mathbb{R}^{n}\right)$ of radius $\beta$, there is a point on the sphere so that the solution with such data will decay arbitrarily slow. In other words, given a time $T>0$, and $\varepsilon>0$, there exists data $u_{0}$ with $\left\|u_{0}\right\|_{2}=\beta$ so that

$$
\frac{\|u(T)\|_{2}}{\left\|u_{0}\right\|_{2}} \geq 1-\varepsilon
$$

Similarly for $B$ given a time $T>0$, and $\varepsilon>0$, there exists data $B_{0}$ with $\left\|B_{0}\right\|_{2}=\gamma$ such that

$$
\frac{\|B(T)\|_{2}}{\left\|B_{0}\right\|_{2}} \geq 1-\varepsilon
$$

Theorem 3. There exist no functions $G(t, \beta)$ and $H(t, \gamma)$ with the following two properties. If $(u, B)$ is a solution to equations (1.耳)-(1.5) with $\delta>0$ and data $\left(u_{0}, B_{0}\right) \in\left(L^{2}\left(\mathbb{R}^{n}\right)\right)^{2}, n=2,3$, then

i) $\|u(t)\|_{2} \leq G\left(t,\left\|u_{0}\right\|_{2}\right)$, and $\|B(t)\|_{2} \leq H\left(t,\left\|B_{0}\right\|_{2}\right)$,

ii) $\lim _{t \rightarrow 0} G(t, \beta)=0$, for all $\beta>0$, and $\lim _{t \rightarrow 0} H(t, \gamma)=0$, for all $\gamma>0$.

Proof. As pointed in Sch86 this lack of uniformity is already present at the level of the heat equation.

The proof of Proposition 2.1 in $S$ ch86] has a gap that will be taken care of in our present work.

Notice that it suffices to show that (5.12) and (5.13) hold. The plan is to choose as initial data a family $\left\{u_{0}^{\alpha}, B_{0}^{\alpha}\right\}$ which satisfy

$$
\left\|u_{0}^{\alpha}\right\|_{2}=\left\|u_{0}\right\|_{2} \quad \text { and } \quad\left\|B_{0}^{\alpha}\right\|_{2}=\left\|B_{0}\right\|_{2} .
$$

Write the solutions in integral form

$$
\begin{aligned}
& u^{\alpha}(x, t)=K * u_{0}^{\alpha}-\int_{0}^{t} K(x-y, t-s) *\left(u^{\alpha} \nabla u^{\alpha}-B^{\alpha} \nabla B^{\alpha}+\nabla p^{\alpha}\right) d s, \\
& B^{\alpha}(x, t)=K * B_{0}^{\alpha}-\int_{0}^{t} K(x-y, t-s) *\left(u^{\alpha} \nabla B^{\alpha}-B^{\alpha} \nabla u^{\alpha}\right) d s,
\end{aligned}
$$

then

$$
\begin{aligned}
& \left\|u^{\alpha}(x, t)\right\|_{2} \geq\left\|K * u_{0}^{\alpha}\right\|_{2}-\int_{0}^{t}\left\|K(x-y, t-s) *\left(u^{\alpha} \nabla u^{\alpha}-B^{\alpha} \nabla B^{\alpha}+\nabla p^{\alpha}\right)\right\|_{2} d s, \\
& \left\|B^{\alpha}(x, t)\right\|_{2} \geq\left\|K * B_{0}^{\alpha}\right\|_{2}-\int_{0}^{t}\left\|K(x-y, t-s) *\left(u^{\alpha} \nabla B^{\alpha}-B^{\alpha} \nabla u^{\alpha}\right)\right\|_{2} d s .
\end{aligned}
$$


The choice of $\left(u_{0}^{\alpha}, B_{0}^{\alpha}\right)$ will be the following

$$
u_{0}^{\alpha}(x)=\alpha^{n / 2} u_{0}(x), \quad B_{0}^{\alpha}(x)=\alpha^{n / 2} B_{0}(x), \quad \text { for } n=2,3 .
$$

Then it is easy to see that $u_{0}^{\alpha}, B_{0}^{\alpha}$ are invariant under $\alpha$ in $L^{2}$ (i.e., Equation (5.14) is satisfied). Hence it is only necessary to show

$$
\frac{\left\|K * u_{0}^{\alpha}\right\|}{\left\|u_{0}\right\|} \geq 1-\varepsilon, \quad \text { and } \quad \frac{\left\|K * B_{0}^{\alpha}\right\|}{\left\|B_{0}\right\|} \geq,
$$

and

$$
\begin{array}{r}
\int_{0}^{t}\left\|K(x-y, t-s) *\left(u^{\alpha} \nabla u^{\alpha}-B^{\alpha} \nabla B^{\alpha}+\nabla p^{\alpha}\right)\right\|_{2} d s<\tilde{\varepsilon} \\
\int_{0}^{t}\left\|K(x-y, t-s) *\left(u^{\alpha} \nabla B^{\alpha}-B^{\alpha} \nabla u^{\alpha}\right)\right\|_{2} d s<\tilde{\varepsilon}
\end{array}
$$

for $\tilde{\varepsilon}$ sufficiently small.

We also note that the data $\left(u_{0}^{\alpha}, B_{0}^{\alpha}\right)$ will yield for the linear part a self-similar solution, that is

$$
\begin{aligned}
K * u_{0}^{\alpha}(x, t) & =\alpha^{n / 2} \tilde{u}\left(\alpha x, \alpha^{2} t\right), \\
K * B_{0}^{\alpha}(x, t) & =\alpha^{n / 2} \tilde{B}\left(\alpha x, \alpha^{2} t\right),
\end{aligned}
$$

Equalities (5.16) follow by uniqueness of the heat equation and since $\alpha^{n / 2} \tilde{u}\left(\alpha x, \alpha^{2} t\right)$ and $K * u_{0}^{\alpha}(x, t)$ are solutions to the heat equation $u_{0}^{\alpha}$. In the same way $\alpha^{n / 2} \tilde{B}\left(\alpha x, \alpha^{2} t\right)$ and $K * B_{0}^{\alpha}(x, t)$ are solutions of the heat equation with data $B_{0}^{\alpha}$. We only will show the proof for the energy of the velocity since the proof for the energy of the magnetic field is almost identical.

We show first that Equation (5.12) holds for the data $u_{0}^{\alpha}$.

$$
\begin{aligned}
\int_{\mathbb{R}^{n}}\left|\tilde{u}^{\alpha}\right|^{2} d x & =\alpha^{n} \int_{\mathbb{R}^{n}}\left|\tilde{u}\left(\alpha x, \alpha^{2} t\right)\right|^{2} d x=\int_{\mathbb{R}^{n}}\left|\tilde{u}\left(y, \alpha^{2} t\right)\right|^{2} d y \\
& =\int_{\mathbb{R}^{n}}\left|\hat{\tilde{u}}\left(\xi, \alpha^{2} t\right)\right|^{2} d \xi=\int_{\mathbb{R}^{n}} e^{-2|\xi|^{2} \alpha^{2} t}\left|\hat{u}_{0}(\xi)\right|^{2} d \xi
\end{aligned}
$$

Next, by Lebesgue Dominated Convergence theorem it follows that for each fixed $t$,

$$
\lim _{\alpha \rightarrow 0} \int_{\mathbb{R}^{n}} e^{-2|\xi|^{2} \alpha^{2} t}\left|\hat{u}_{0}(\xi)\right|^{2} d \xi=\int_{\mathbb{R}^{n}}\left|\hat{u}_{0}\right|^{2} d \xi
$$

Hence

$$
\lim _{\alpha \rightarrow 0} \frac{\left\|\tilde{u}^{\alpha}(\cdot, t)\right\|_{2}^{2}}{\left\|u_{0}(\cdot)\right\|_{2}^{2}}=1
$$

Now it is necessary to show

$$
\lim _{\alpha \rightarrow 0} \int_{0}^{t}\left\|K(x-y, t-s) *\left(u^{\alpha} \nabla u^{\alpha}-B^{\alpha} \nabla B^{\alpha}+\nabla p^{\alpha}\right)\right\|_{2} d s=0,
$$


Hence we analyze

$$
\begin{aligned}
\int_{0}^{T} \| K(x-y, t-s) & *\left(u^{\alpha} \nabla u^{\alpha}-B^{\alpha} \nabla B^{\alpha}+\nabla p^{\alpha}\right) \|_{2} d s \\
& \leq \int_{0}^{T}\|\nabla K(t-s)\|_{2}\left(\left\|u^{\alpha} u^{\alpha}\right\|_{2}+\left\|B^{\alpha} B^{\alpha}\right\|_{2}+\left\|p^{\alpha}\right\|_{2}\right) d s \\
& \leq C \int_{0}^{T} \frac{1}{(t-s)^{1 / 2}}\left(\left\|u^{\alpha} u^{\alpha}\right\|_{2}+\left\|B^{\alpha} B^{\alpha}\right\|_{2}\right) d s
\end{aligned}
$$

Here we used that

$$
\left\|p^{\alpha}\right\|_{2} \leq C\left(\left\|u^{\alpha} u^{\alpha}\right\|_{2}+\left\|B^{\alpha} B^{\alpha}\right\|_{2}\right) .
$$

We can suppose then that we have chosen $u_{0}$ and $B_{0}$ to be in $H^{1}$. Now observe that for $n=3$

$$
\left\|u^{\alpha} u^{\alpha}\right\|_{2} \leq C\left\|\nabla u^{\alpha}\right\|_{2}^{3 / 2}, \quad\left\|B^{\alpha} B^{\alpha}\right\| \leq C\left\|\nabla B^{\alpha}\right\|_{2}^{3 / 2}
$$

and for $n=2$

$$
\left\|u^{\alpha} u^{\alpha}\right\|_{2} \leq C\left\|\nabla u^{\alpha}\right\|_{2}, \quad\left\|B^{\alpha} B^{\alpha}\right\|_{2} \leq C\left\|\nabla B^{\alpha}\right\|_{2} .
$$

Also

$$
\begin{aligned}
\|\nabla u\|_{2}+\|\nabla B\|_{2} & \leq C\left(\|\nabla u\|_{2}^{2}+\|\nabla B\|_{2}^{2}\right)^{1 / 2} \text { and } \\
\|\nabla u\|_{2}^{3 / 2}+\|\nabla B\|_{2}^{3 / 2} & \leq C\left(\|\nabla u\|_{2}^{2}+\|\nabla B\|_{2}^{2}\right)^{3 / 4} .
\end{aligned}
$$

Thus to bound the right hand side of (5.19) we need to estimate

$$
\varphi(t)=\left\|\nabla u^{\alpha}\right\|_{2}^{2}+\left\|\nabla B^{\alpha}\right\|_{2}^{2} .
$$

Note first that if we choose $\left(u_{0}, B_{0}\right) \in H_{1} \times H_{1}$ will yield that $\left\|\nabla u^{\alpha_{0}}\right\|_{2},\left\|\nabla B^{\alpha_{0}}\right\|_{2}$ are arbitrarily small if $\alpha \ll 1$. This follows since

$$
\begin{aligned}
\varphi(0) & =\varphi_{0}=\int\left|\nabla u_{0}^{\alpha}\right|^{2} d x+\int\left|\nabla B_{0}^{\alpha}\right|^{2} d x \\
& =\int \alpha^{4}\left|\nabla u_{0}(\alpha x)\right|^{2} d x+\int \alpha^{4}\left|\nabla B_{0}(\alpha x)\right|^{2} d x \\
& =\alpha^{2}\left(\int\left|\nabla u_{0}(x)\right|^{2} d x+\int\left|\nabla B_{0}(x)\right|^{2} d x\right) \\
& =C \alpha^{2} .
\end{aligned}
$$

In order to estimate $\left\|\nabla u^{\alpha}\right\|_{2}$ and $\left\|\nabla B^{\alpha}\right\|_{2}$ we use Prodi's inequality. We consider two cases:

Case 1: $(n=2)$. By Prodi,

$$
\frac{d \varphi}{d t} \leq C \varphi^{2} \Rightarrow \frac{d \varphi}{\varphi} \leq C \varphi d t \quad \Rightarrow \quad \ln \left(\frac{\varphi(t)}{\varphi_{0}^{\alpha}}\right) \leq C \int_{0}^{t} \varphi(s) d s,
$$

which implies

$$
\begin{aligned}
\varphi(t) & \leq \varphi_{0}^{\alpha} e^{C \int_{0}^{t} \varphi(s) d s} \leq \varphi_{0}^{\alpha} e^{C\left(\left\|u_{0}\right\|_{2}^{2}+\left\|B_{0}\right\|_{2}^{2}\right)} \leq \varphi_{0}^{\alpha} \\
& \leq C \alpha^{2} .
\end{aligned}
$$


Hence,

$$
\int_{0}^{t}\left\|K(x-y, t-s) *\left(u^{\alpha} \nabla u^{\alpha}-B^{\alpha} \nabla B^{\alpha}+\nabla p^{\alpha}\right)\right\|_{2} d s \leq C T^{1 / 2} \alpha^{2},
$$

and thus (5.18) follows when $n=2$.

By 5.18 and (5.17) the theorem follows for the velocity in two dimensions.

Case 2: $(n=3)$. By Prodi,

$$
\frac{d \varphi}{d t} \leq C \varphi^{3} \Rightarrow \frac{d \varphi}{\varphi^{2}} \leq C \varphi d t .
$$

Integrating,

$$
\begin{aligned}
\frac{1}{\varphi_{0}}-\frac{1}{\varphi(t)} & \leq C \int_{0}^{t} \varphi(s) d s \\
& \leq C\left(\left\|u_{0}\right\|_{2}^{2}+\left\|B_{0}\right\|_{2}^{2}\right)=C .
\end{aligned}
$$

Solving for $\varphi(t)$ we get

$$
\varphi(t) \leq \frac{\varphi_{0}}{1-C_{0} \varphi_{0}}=\frac{C \alpha^{2}}{1-\tilde{C} \alpha^{2}} \leq 2 C \alpha^{2},
$$

where we have chosen $\alpha$ very small, say $\tilde{C} \alpha^{2} \leq \frac{1}{2}$, to make the last inequality true. Hence, for the case $n=3$, the expression (5.18) is negligible too. Combining (5.18) and (5.17) yields the conclusion of the theorem for the velocity in three dimensions.

The estimate for the magnetic field in 2 and 3 dimensions follows in an analogous fashion. This completes the proof of the theorem.

\section{KATO'S ESTimates}

In this section we show that by some simple modification Kato's pioneering work on $L^{p}$ decay for Navier-Stokes equations [Kat84 holds for the MHD equations with magnetic diffusion. The main difference is that his approximating solutions will be replaced by the corresponding ones from MHD. Thus, rewrite the MHD equations as follows

$$
\partial_{t} v+A v+F(v)=0
$$

where $A=\left(A_{1}, A_{2}\right)=-P(\Delta, \delta \Delta), v=(u, B), F(v)=F(v, v)=\left(F_{1}(v, v), F_{2}(v, v)\right)$, and

$$
\begin{aligned}
& F_{1}(u, w, B, D)=P(u \nabla w)-P(B \nabla D) \\
& F_{2}(u, w, B, D)=P(u \nabla B)-P(B \nabla u) .
\end{aligned}
$$

Here $P$ is the orthogonal projection of $L^{2}$ onto the subspace $P L^{2}$, which denotes the collection of divergence-free elements of $L^{2}$.

Following Kato

$$
\begin{aligned}
& u_{n+1}=u_{0}(t)+G_{1}\left(u_{n}, B_{n}\right), \\
& B_{n+1}=B_{0}(t)+G_{2}\left(u_{n}, B_{n}\right),
\end{aligned}
$$


where

$$
G_{i}(u, B)=-\int_{0}^{t} e^{-(t-s) A_{i}} F_{i}(u(s), B(s)) d s
$$

and

$$
\left(u_{0}(\cdot, t), B_{0}(\cdot, t)\right)=\left(e^{-t A_{1}} u_{0}(x), e^{-t A_{2}} B_{0}(x)\right) .
$$

Using these expressions appropriately in theorems 1,2, and 3 in Kato's paper Kat84 will yield the same results for the MHD equations.

We want to show how these results can be used to extend the decay results for the MHD equations in two dimensions when combined with our $L^{2}$ results. We recall, for easy reference, Kato's first two theorems. In this case $u$ stands for the solution to the Navier-Stokes equations

$$
u_{t}+u \cdot \nabla u+\nabla p=\Delta u, \quad \operatorname{div} u=0 .
$$

Theorem (Kato 1). Let the initial data $a \in P L^{m}$. Then there is $T>0$ and a unique solution $u$ such that

$$
\begin{array}{rlrl}
t^{(1-m / q) / 2} u & \in B C\left([0, T) ; P L^{q}\right) & & \text { for } m \leq q \leq \infty, \\
t^{1-m / 2 q} \nabla u & \in B C\left([0, T) ; P L^{q}\right) & \text { for } m \leq q<\infty,
\end{array}
$$

both with values zero at $t=0$ except for $q=m$ in (1.1), in which $u(0)=a$. Moreover, $u$ has the additional property

$$
u \in L^{r}\left(\left(0, T_{1}\right) ; P L^{q}\right) \quad \text { with } 1 / r=(1-m / q) / 2, \quad m<q<m^{2} /(m-2),
$$

with some $0<T_{1} \leq T$.

Theorem (Kato 2). There is $\lambda>0$ such that if $\|a\|_{m} \leq \lambda$, then the solution $u$ in Theorem (1) is global, i.e. we may take $T=T_{1}=\infty$. In particular, $\|u(t)\|_{q}$ decays like $t^{-(1-m / q) / 2}$ as $t \rightarrow \infty$, including $q=\infty$, and $\|\nabla u(t)\|_{q}$ decays like $t^{-(1-m / 2 q)}$, including $q=m$.

As stated before usingg $\left(u_{n+1}, B_{n+1}\right)$ as defined above and following Kato's proof with straightforward modifications yields

Theorem 4. Let $n=2,3$. Suppose $\left(u_{0}, B_{0}\right) \in\left(P L^{p} \cap P L^{n}\left(\mathbb{R}^{n}\right)\right)^{2}$, where $1<p<$ $n$. There exists $\lambda_{1}>0$ such that if $\left\|u_{0}\right\|_{n} \leq \lambda_{1}$ and $\left\|B_{0}\right\|_{n} \leq \lambda_{1}$, then the solution to the MHD equations with $\delta>0$ is global and for any finite $q \geq p$

$$
t^{(n / p-n / q) / 2}(u, B) \quad \text { and } \quad t^{(n / p-n / q+1) / 2}(\nabla u, \nabla B) \in B C\left([1, \infty] ; P L^{q}\right)^{2} .
$$

Combining the results of Theorems 1 and 1 in the two dimensional case yields the following improved decay for the solutions to the MHD equations with $\delta>0$.

Corollary 5. There is $\lambda>0$ such that for $\left\|u_{0}\right\|_{2} \leq \lambda$ the global solution of the equation (6.1) for $q \geq m$, and for $2 \leq r \leq q$

$$
\lim _{t \rightarrow \infty} t^{\frac{r-2}{2 r}}\|(u, B)\|_{r}=0
$$

Proof. It follows by interpolating $L^{r}$ between $L^{2}$ and $L^{q}$ and using the decay rates of the solutions corresponding to those Sobolev spaces. 


\section{Appendix}

Proposition 3. Let $(u, B)$ be a solution to the MHD equations (1.7)-(1.5). Assume the initial data $u_{0}, B_{0}$ is in $L^{1}\left(\mathbb{R}^{3}\right) \cap L^{2}\left(\mathbb{R}^{3}\right)$. Then

$$
|\hat{u}(t)| \leq C\left(1+\frac{1}{|\xi|}\right)
$$

where $C$ is a constant.

Proof. We start by taking the Fourier transform of Equation (1.2)

$$
\hat{u}_{t}+\widehat{u \cdot \nabla u}-\widehat{B \cdot \nabla B}+\widehat{\nabla p}=-|\xi|^{2} \hat{u} .
$$

Let us define

$$
H(\xi, t)=\widehat{u \cdot \nabla u}-\widehat{B \cdot \nabla B}+\widehat{\nabla p}
$$

Then $\hat{u}_{t}+|\xi|^{2} \hat{u}=-H(\xi, t)$, and this equation can be integrated using the method of integrating factor to get

$$
\hat{u}(t)=\hat{u}(0) e^{-|\xi|^{2} t}-\int_{0}^{t} H(\xi, s) e^{-|\xi|^{2}(t-s)} d s .
$$

Then,

$$
|\hat{u}(t)| \leq\left|\hat{u}_{0}\right|+\int_{0}^{t}|H(\xi, s)| e^{-|\xi|^{2}(t-s)} d s .
$$

To bound $|H(\xi, s)|$, let us first bound $|\widehat{\nabla p}|$. For this, let us take the divergence operator in Equation (1.2) which yields

$$
\Delta p=\sum_{k, j} \frac{\partial^{2}}{\partial x^{k} \partial x^{j}}\left(B^{j} B^{k}-u^{j} u^{k}\right) .
$$

It follows that

$$
\begin{aligned}
|\widehat{\nabla p}| & =|\xi||\hat{p}| \leq \sum_{k, j} \frac{\left|\xi^{k} \xi^{j}\right|}{|\xi|}\left(\left|\widehat{B^{j} B^{k}}\right|+\left|\widehat{u^{j} u^{k}}\right|\right) \\
& \leq|\xi| \sum_{k, j}\left(\left|\widehat{B^{j} B^{k}}\right|+\left|\widehat{u^{j} u^{k}}\right|\right)
\end{aligned}
$$

Hence

$$
\begin{aligned}
|H(\xi, s)| & \leq C|\xi| \sum_{j, k}\left(\left|\widehat{u^{j} u^{k}}\right|+\left|\widehat{B^{j} B^{k}}\right|\right) \\
& \leq C|\xi|\left(\left\|u_{0}\right\|_{2}^{2}+\left\|B_{0}\right\|_{2}^{2}\right) \leq C|\xi|
\end{aligned}
$$

Since $\left|\hat{u}_{0}\right| \leq\left\|u_{0}\right\|_{1}=C$, it follows that

$$
\begin{aligned}
|\hat{u}| & \leq\left|\hat{u}_{0}\right|+C|\xi| \int_{0}^{t} e^{-|\xi|^{2}(t-s)} d s \leq C+\frac{C}{|\xi|}\left(1-e^{-|\xi|^{2} t}\right) \\
& \leq C+\frac{C}{|\xi|}=C\left(1+\frac{1}{|\xi|}\right)
\end{aligned}
$$

which finishes the proof. 


\section{REFERENCES}

[Cha81] S. Chandrasekhar. Hydrodynamic and Hydromagnetic Stability. Dover Publications, New York, 1981.

[CKN82] L. Caffarelli, R. Kohn, and L. Nirenberg. Partial regularity of suitable weak solutions of the Navier-Stokes equations. Comm. Pure Appl. Math., 35(6):771-831, 1982.

[Cow76] T. Cowling. Magnetohydrodynamics. Monographs on astronomical subjects. Hilger, 2 edition, 1976.

[HX05a] Cheng He and Zhouping Xin. On the regularity of weak solutions to the magnetohydrodynamic equations. J. Differential Equations, 213(2):235-254, 2005.

[HX05b] Cheng He and Zhouping Xin. Partial regularity of suitable weak solutions to the incompressible magnetohydrodynamic equations. J. Funct. Anal., 227(1):113-152, 2005.

[Kat84] Tosio Kato. Strong $L^{p}$-solutions of the Navier-Stokes equation in $\mathbb{R}^{m}$, with applications to weak solutions. Math. Z., 187(4):471-480, 1984.

[Kim02] Sangjeong Kim. Gevrey class regularity of the magnetohydrodynamics equations. ANZIAM J., 43(3):397-408, 2002.

[Koz87] Hideo Kozono. On the energy decay of a weak solution of the MHD equations in a three-dimensional exterior domain. Hokkaido Math. J., 16(2):151-166, 1987.

[Ler34] J. Leray. Sur le mouvement d'un liquide visquex emplissant l'espace. Acta Mathematica, 63:193-248, 1934.

[LLP84] L.D. Landau, E.M. Lifshitz, and L.P. Pitaevskii. Electrodynamics of Continuous Media, volume 8 of Course of theoretical physics. Butterworth-Heinemann, 2 edition, 1984.

[MS89] Satish D. Mohgaonkar and R. V. Saraykar. $L^{2}$-decay for solutions of the magnetohydrodynamic equations. J. Math. Phys. Sci., 23(1):35-55, 1989.

[ORS97] Takayoshi Ogawa, Shubha V. Rajopadhye, and Maria E. Schonbek. Energy decay for a weak solution of the Navier-Stokes equation with slowly varying external forces. $J$. Funct. Anal., 144(2):325-358, 1997.

[OT00] Marcel Oliver and Edriss S. Titi. Remark on the rate of decay of higher order derivatives for solutions to the Navier-Stokes equations in $\mathbf{R}^{n}$. J. Funct. Anal., 172(1):1-18, 2000.

[Sch85] Maria Elena Schonbek. $L^{2}$ decay for weak solutions of the Navier-Stokes equations. Arch. Rational Mech. Anal., 88(3):209-222, 1985.

[Sch86] Maria E. Schonbek. Large time behaviour of solutions to the Navier-Stokes equations. Comm. Partial Differential Equations, 11(7):733-763, 1986.

[SSS96] M. E. Schonbek, T. P. Schonbek, and Endre Süli. Large-time behaviour of solutions to the magnetohydrodynamics equations. Math. Ann., 304(4):717-756, 1996.

[Wu02] J. Wu. Bounds and new approaches for the 3D MHD equations. J. Nonlinear Sci., 12(4):395-413, 2002.

Dept. Of Mathematics, UCSC, CA 95064

E-mail address: ragapito@math.ucsc.edu

$U R L:$ http://math.ucsc.edu/ ragapito/

E-mail address: schonbek@math.ucsc.edu

URL: http://math.ucsc.edu/ schonbek/ 